\title{
Long exposure photography implementation in undergraduate physics laboratories
}

\section{Aplicación de la fotografía con largo tiempo de exposición en los laboratorios de enseñanza de la física}

\author{
H. H. Cerecedo-Núñez $\left.{ }^{*}, A\right)$, M. A. Carrillo-Bernal, P. E. Mancera-Piña, P. Padilla-Sosa(A) \\ Laboratorio de Óptica Aplicada, Departamento de Física, Facultad de Física e Inteligencia Artificial, Universidad \\ Veracruzana, Xalapa, Veracruz, México.

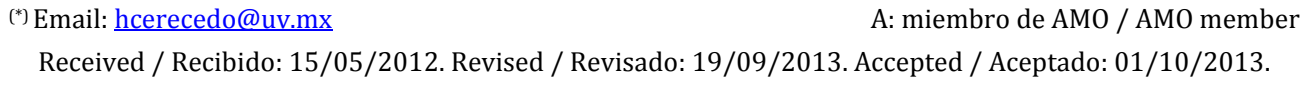

\begin{abstract}
:
This paper reviews the operation of the long exposure photography. We investigate the application of this photographic technique in experiments carried out in physics teaching, in university laboratories. We developed an example, which indicate the potential of this technique in quantitative measurements.
\end{abstract}

Key words: Teaching Methods, Photography, Newtonian Mechanics, Optical Instruments, Optical Sensors.

\section{RESUMEN:}

En este trabajo se revisa el funcionamiento de la fotografía digital, de largos tiempos de exposición. Se investiga la aplicación de esta técnica fotográfica en experimentos que se desarrollan en los laboratorios universitarios de enseñanza de la física. Se desarrolla un ejemplo en el cual se observa el posible potencial de esta técnica en mediciones cuantitativas.

Palabras clave: Métodos de Enseñanza, Fotografía, Mecánica Newtoniana, Instrumentos Ópticos, Sensores Ópticos.

\section{REFERENCES AND LINKS / REFERENCIAS Y ENLACES}

[1]. H. Keppler, The Asahi Pentax Way, Focal Press, $9^{\text {th }}$ Edition (1976).

[2]. B. Peterson, Understanding Exposure, $3^{\text {rd }}$ Edition, Amphoto Books, Kindle Edition (2010).

[3]. B. Long, Complete Digital Photography, $6^{\text {th }}$ Edition, Course Technology CENGAGE Learning, Kindle Edition (2013).

[4]. S. Kelby, The Digital Photography Book, Peachpit Press (2007).

[5]. "Joseph Nicéphore Niépce", http://es.wikipedia.org/wiki/Joseph_Nic\%C3\%A9phore_Ni\%C3\%A9pce (Visited: Sept. 8, 2013).

[6]. "El material fotosensible", http://www.digitalfotored.com/fotografia/matfotosensible.htm (Visited: Sept. 8, 2013).

[7]. Nicéphore Niépce camera, c. 1820-1830, Exhibit in the Musée Nicéphore Niépce, 28 Quai Messageries, Saône-et-Loire (France),

https://fr.m.wikipedia.org/wiki/Fichier:Nic\%C3\%A9phore Ni\%C3\%A9pce camera, c. 18201830 - Mus\%C3\%A9e Nic\%C3\%A9phore Ni\%C3\%A9pce - DSC06018.JPG, (Visited: Sept 8, 2013).

[8] View from the Window at Le Gras, Joseph Nicéphore Niépce. Rebecca A. Moss, Coordinator of Visual Resources and Digital Content Library. College of Liberal Arts Office of Information Technology, University of Minnesota, 
http://en.wikipedia.org/wiki/File:View from the Window at Le Gras, Joseph Nic\%C3\%A9phore Ni\%C3\%A9pce.jpg, (Visited: Sept. 8, 2013).

[9]. "Daguerre y el daguerrotipo", http://www.digitalfotored.com/fotografia/daguerrotipo.htm, (Visited: Sept. 8, 2013).

[10]. "La época del Daguerrotipo", http://www.digitalfotored.com/fotografia/epocadaguerre.htm, (Visited: Sept. 8, 2013).

[11]. "Eadweard Muybridge", http://en.wikipedia.org/wiki/Eadweard Muybridge (Visited: Sept. 8, 2013).

[12]. Panasonic Lumix DMC-FZ5, Operating Instructions, VQT0Q09, Matsushita Electric Industrial Co., Ltd. (2005).

[13]. C. Kittel, W. D. Knight, M. A. Ruderman. Mechanics, Berkeley Physics Course, Vol. 1, Chap. 7, "The harmonic oscillator" (1968).

[14]. GIMP, GNU Image Manipulation Program, http://www.gimp.org/ (Visited: Sept. 8, 2013).

[15]. Inkscape, http://inkscape.org/index.php?lang=es (Visited: Sept. 8, 2013).

[16]. D. C. Baird, Experimentation: An Introduction to Measurement and Experiment Design, Prentice-Hall (1991).

[17]. Aceleración de la gravedad para la ciudad de Xalapa, Enríquez, Veracruz. http://www.metas.com.mx/utilerias/calculoacelgravedad.php (Visited: April 5, 2013)

[18]. Aceleración de la gravedad para la ciudad de Xalapa, Enríquez, Veracruz. http://www.physicsclassroom.com/class/circles/u6l3e.cfm (Visited: April 5, 2013)

[19]. M. A. Carrillo-Bernal, P. E. Mancera-Piña, H. H. Cerecedo-Núñez, P. Padilla-Sosa, H. N. Núñez-Yépez, A. L. Salas-Brito, "The ellipse in parabolic motion: an undergraduate experiment", in review, Am. J. Phys. (2013).

[20]. E. Hecht, Optics, $2^{\text {nd }}$ Edition, Chapter 9, Addison-Wesley (1987).

\section{Introducción}

En décadas pasadas era muy conocida la técnica fotográfica de largo tiempo de exposición con cámaras tipo réflex, profesionales. Sin embargo, dado la complejidad en la preparación de las tomas, en el revelado, así como en el tiempo de procesamiento, parecía complicado implementarlo en una práctica de unas cuantas horas de laboratorio. En años más recientes, las cámaras digitales son de fácil acceso. Un poco menos, las cámaras digitales semi-profesionales y profesionales, con funciones diversas y espectaculares. Hoy en día, con las cámaras digitales, es muy rápido preparar y procesar una exposición [1-4]. Con ello es factible emplearlas como herramienta de medición en los laboratorios de física.

En este artículo documentamos la fotografía de largo tiempo de exposición enfocándonos en mediciones que se pueden realizar en los laboratorios de enseñanza de la física. Para ello, en la sección 2 se da una descripción breve de los antecedentes históricos de la fotografía de largo tiempo de exposición; en la sección 3 se da también una breve explicación técnica del tiempo de exposición en las cámaras fotográficas; en la sección 4 se discuten los resultados preliminares para llevar a cabo pruebas cuantitativas, y finalmente, en la sección 5 se explica un ejemplo cuantitativo en el uso de esta técnica.

Este documento está dirigido a estudiantes universitarios o profesores de nivel superior que deseen implementar una herramienta de apoyo en mediciones relacionadas con la dinámica o la cinemática de fenómenos físicos.

\section{Antecedentes históricos}

La historia de la fotografía es muy extensa. Nos enfocaremos en el desarrollo del control de los tiempos de exposición de las cámaras. La primera cámara formal fue inventada por Nicephore Niépce [5,6], alrededor de 1825. La fotografía más antigua registrada es aproximadamente del año 1826 (ver Fig. 1). Cabe decir que tanto antes como ahora el tiempo de exposición es el tiempo en el que se tomaba la 

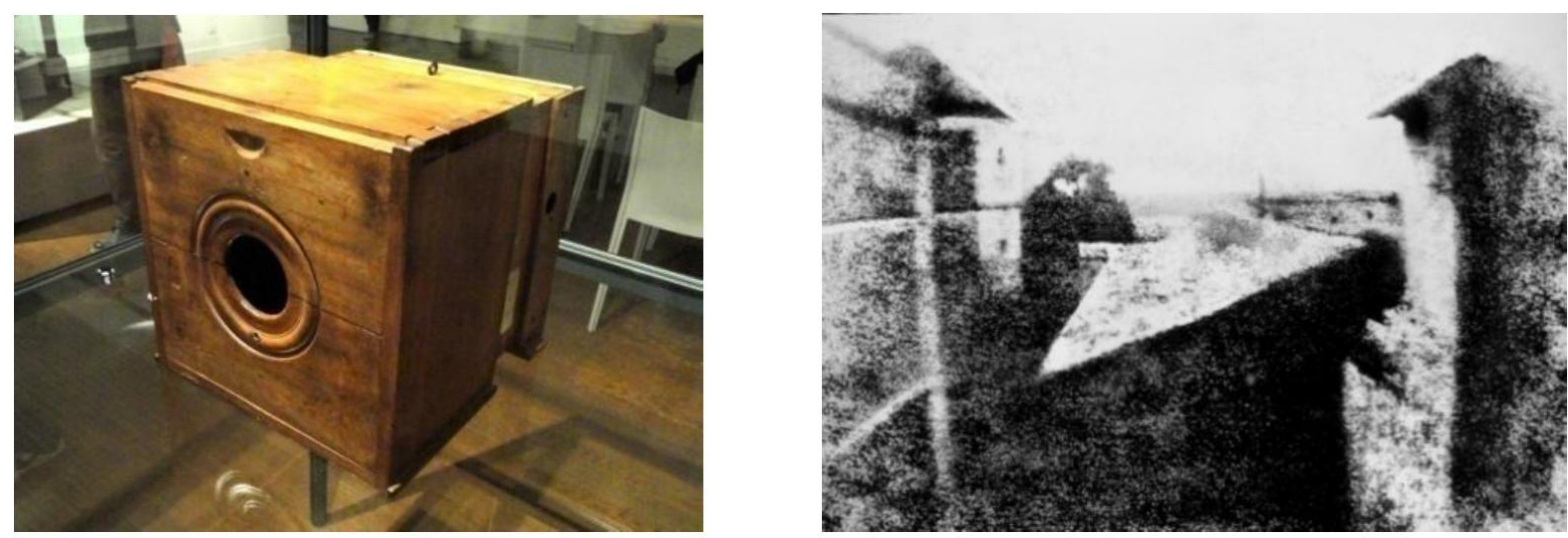

Fig. 1. Primera cámara fotográfica [7] y primera fotografía ambos por Joseph Nicéphore Niépce [8].

fotografía; pero antes era el tiempo necesario para que la fotografía quedara grabada en la placa (el promedio de tiempo de exposición era de media hora), por lo que muchas fotos quedaban borrosas si el objeto o la cámara se movían, lo que implicaba que mientras menor fuera el tiempo necesario, era mejor.

En 1842 la fotografía evolucionó, pues gracias a los avances en la composición química de las placas, se logró reducir el tiempo de exposición de 30 minutos a un intervalo entre 30 y 40 segundos.

Entre los primeros pasos que tuvo la fotografía científica esta el que desarrollaron personas como Soleil (Jean-Baptiste François Soleil) quien ingenió un microscopiodaguerrotipo en 1839 (daguerrotipo se refiere a la primera cámara que existió [9], así que el microscopio-daguerrotipo fue el primer microscopio con cámara); en 1840 el físico John Wiliam Draper tomó una fotografía de la Luna por lo que se le considera el primer fotógrafo de astros y, cinco años más tarde, Fizeau y Foucault fotografiaron el Sol [10].

El primer experimento formal de la fotografía con tiempo de exposición controlada fue el que realizó Eadweard Muybridge [11]. Este experimento surgió en 1872, cuando debido a confrontaciones entre aficionados a las carreras de caballos se buscó idear un arreglo que permitiera ver si había un momento en el que los 4 cascos del caballo permanecían en el aire; en aquella época, un empresario, Leland Stanford apoyaba esta idea, mientras que un sector presidido por James Keene sostenía lo contrario, por lo que Stanford ideó un experimento. Éste fue encargado al científico Eadweard Muybridge y consistía en captar con una cámara imágenes de un caballo en movimiento durante las distintas etapas de su galope, el trote del caballo "Occident" tuvo una velocidad de $35 \mathrm{~km} / \mathrm{h}$; Muybridge colocó sábanas blancas a lo largo de una pista e intentó fotografiar a "Occident", pero no tuvo buenos resultados debido a que el tiempo de exposición exigía varios segundos para obtener una buena calidad de imagen. La solución a este problema apareció cuando en 1878 Muybridge encontró una manera para producir nítidos negativos de imágenes, y así obtuvo una clara silueta de "Occident" (ver Fig. 2), en la que se observaba que había un instante en el que tenía sus cuatro cascos al aire, lo que le daba la razón a Stanford.

Sin embargo, con este método no conseguía verse el caballo en sí, solo su silueta, por lo que Muybridge no dejo esta cuestión de lado y se dio cuenta de que sus primeros intentos habían fallado debido a que el tiempo de exposición en que tomaba las fotos eran muy largos a comparación del instante que trataba de capturar, por lo que no paró de experimentar e ideó un obturador mecánico que estaba hecho con dos pares de hojas de madera las cuales deslizaban por las ranuras de un marco y dejaban ver una abertura de $20 \mathrm{~cm}$ por la cual penetraba la luz. Fue con este tipo de obturador que Muybridge logró conseguir un tiempo de exposición de tan solo $1 / 500$ segundos. Una última sesión de fotos del recorrido de una yegua (Sally Gardner), proporcionó una serie de 


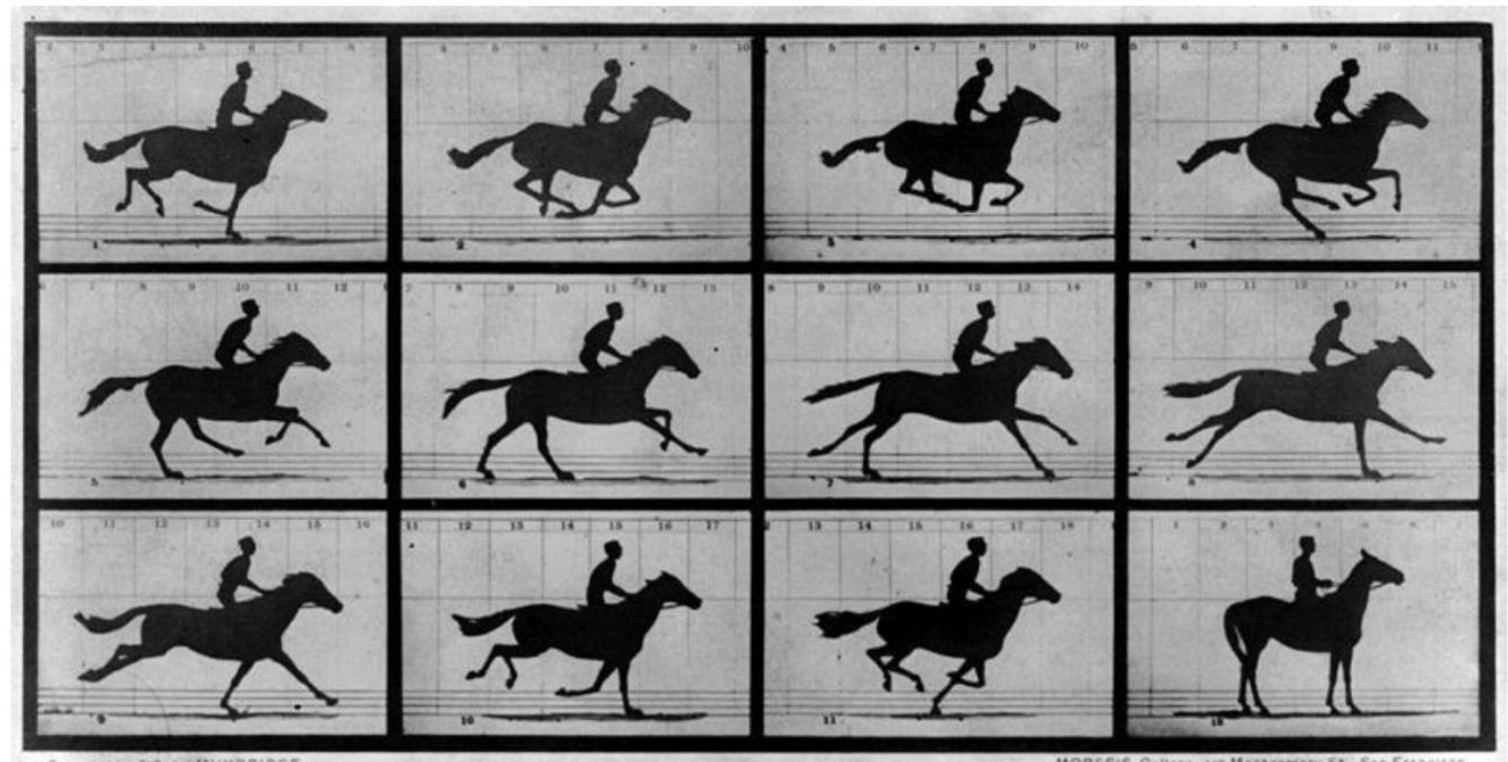

Fig. 2. "El caballo en movimiento”, fotografías de Muybridge [11].

12 fotografías que se realizaron en un medio de segundo, en las fotografías se pudieron ver todas las posiciones que adoptaba la yegua y que no eran perceptibles al ojo humano.

El médico francés Etienne Jules Marey continuó la investigación de Muybridge, mejorando su arreglo experimental y así logró obtener el desarrollo del movimiento en tan sólo un "cliché". En la actualidad, el perfeccionamiento de la fotografía en la era digital nos permite controlar el tiempo de exposición fácilmente, resultando así una herramienta muy útil, pues permite capturar el movimiento de un objetivo con precisión; sin embargo, sus aplicaciones actuales tienen un enfoque más artístico, hay muchas exposiciones de galerías de imágenes fotografiadas con esta técnica.

\section{Fotografía de larga exposición}

La fotografía con tiempo de exposición es una técnica fotográfica altamente usada en el arte y la astronomía, que consiste en controlar el tiempo que el obturador de la cámara recibirá la luz del medio [1-4].

La apertura, número $f$, es una apertura que podemos ajustar mediante el diafragma (una estructura formada con varias láminas finas interpuesta entre las lentes y la cámara) regulando la cantidad de luz que entra al sistema
(Fig. 3); junto con la velocidad de obturación, regula el grado de exposición a la luz del sensor determinando el valor de exposición.

La velocidad de obturación se refiere al inverso del tiempo de exposición. El tiempo de exposición es el tiempo en que la luz alcanza el sensor digital, es decir, el tiempo en el que se hace la fotografía. El obturador es una cortinilla que se abre al momento de disparar, limitando el tiempo en que la luz incide a la cámara.

Dependiendo de la cámara los tiempos de exposición pueden variar yendo desde segundos, para pobres condiciones de luz, hasta milésimas de segundo, para fotos rápidas; algunos de los tiempos de exposición más comunes son (en segundos): $4,2,1,1 / 2,1 / 4,1 / 8,1 / 15,1 / 30$, $1 / 60,1 / 125,1 / 250,1 / 500,1 / 1000$ y $1 / 2000$. También existe la posibilidad, con el equipo adecuado, de extender el tiempo de exposición indefinidamente, llegando incluso a durar por meses.

La escala de tiempos de exposición funciona mediante una sucesión de pasos, tal que cada paso implica la incidencia del doble de la cantidad de luz que en un paso anterior. La exposición es igual al producto de la iluminancia (cantidad de flujo luminoso por unidad de área) por el tiempo, por lo que se mide en lux por segundo, representando así la combinación de la iluminación recibida y el tiempo. 

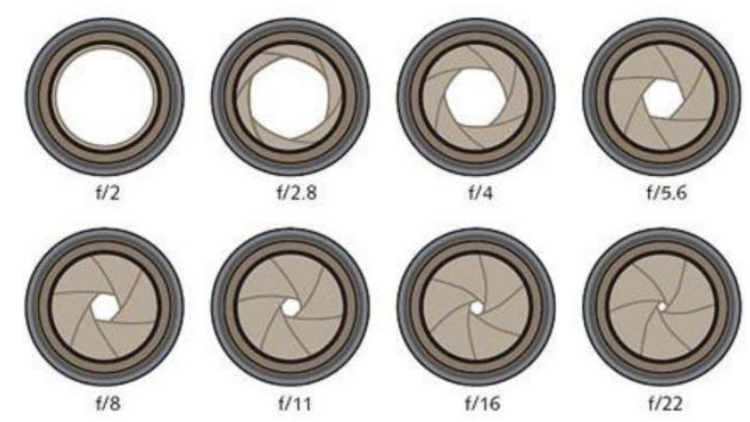

Fig. 3. Aberturas del obturador para diferentes valores del número $f$.

Es importante aclarar que no cualquier cámara es capaz de realizar fotografías con exposición controlada, en este proyecto se utilizó una cámara con sensor digital (tipo CCD).También es importante mencionar que los sensores digitales tienen una resolución en función del número de pixeles.

\section{Pruebas preliminares}

Para la investigación de la fotografía de larga exposición se ocuparon: una cámara digital, un tripié para evitar la trepidación (errores por movimiento) y diversas fuentes luminosas (para realizar distintas pruebas). Para cerciorarnos de la mecánica de este tipo de fotografía, las pruebas preliminares se hicieron a tiempos distintos y con distintas fuentes de luz (Fig 4).

El efecto obtenido mediante esta técnica fotográfica es sin duda atractivo para la vista, pero más que degustar a la gente, el objetivo respecto a este tipo de fotografía es el de proponer un uso de ésta para ayudar en el estudio de la física. En general, para todas las fotografías que se presentan, se empleo una cámara Panasonic DMC-FZ5PL [12], con una velocidades de disparo que va de $1 / 2000$ a 8 segundos y aberturas de: $f / 2.8, f / 3.2, f / 3.6$, $f / 4.0, f / 4.5, f / 5.0, f / 5.6, f / 6.3, f / 7.1$ y $f / 8.0$.

Habiéndonos familiarizado con este tipo de fotografía llevamos a cabo otra serie de sesiones para observar otros fenómenos. La Fig. 5 muestra una serie de fotografías obtenidas al encender la luz de un teléfono celular convencional, obsérvese como con cada paso en la exposición, pareciera ir aumentando su
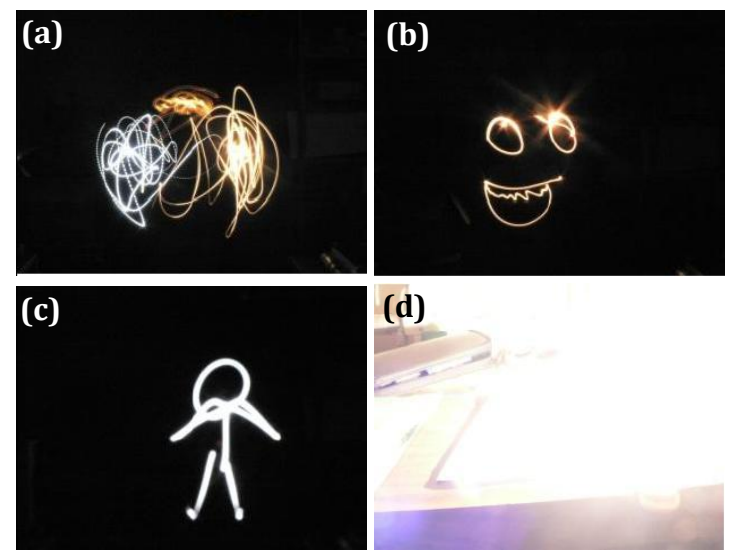

(d)

Fig. 4. Pruebas preliminares: las imágenes se realizaron con un tiempo de exposición de 8 segundos y una abertura de $f / 8.0$. (a) Tres lámparas en movimiento, (b) una lámpara, dibujando en el aire la silueta de una cara, (c) una lámpara de celular, dibujando el clásico "stickman" y (d) un foco sin movimiento. En este caso la imagen recibe demasiada luz por el tiempo de exposición y ésta se satura.

intensidad poco a poco, cuando en realidad el celular estuvo encendido todo el tiempo; también se pueden observar ciertos detalles en la superficie en la que está, una hoja de papel blanca, las partes deformadas sobre la superficie de la hoja van haciéndose más y más evidentes. La explicación anterior se debe a que, a medida que el tiempo de exposición de cada imagen aumenta, más luz es captada por el sensor de la cámara y por ello aumenta la definición de cada imagen, hasta que llega el momento en que la exposición es suficiente y posteriormente se puede saturar.

Después de haber realizado las primeras pruebas con este tipo de fotografía se observa la posibilidad de que puede ser útil en el laboratorio de física, por ejemplo, para el estudio de la cinemática de los cuerpos. Como ejemplo concreto, implementaremos esta técnica en un péndulo simple, pues debido a su movimiento es posible estudiarlo de manera muy completa.

\section{Implementación: El uso del péndulo como instrumento gravimétrico}

Desde Galileo, el péndulo ha sido largamente estudiado y hoy en día se sabe que la masa no es determinante en su movimiento y que el tiempo que tarda una oscilación completa está dado por: 


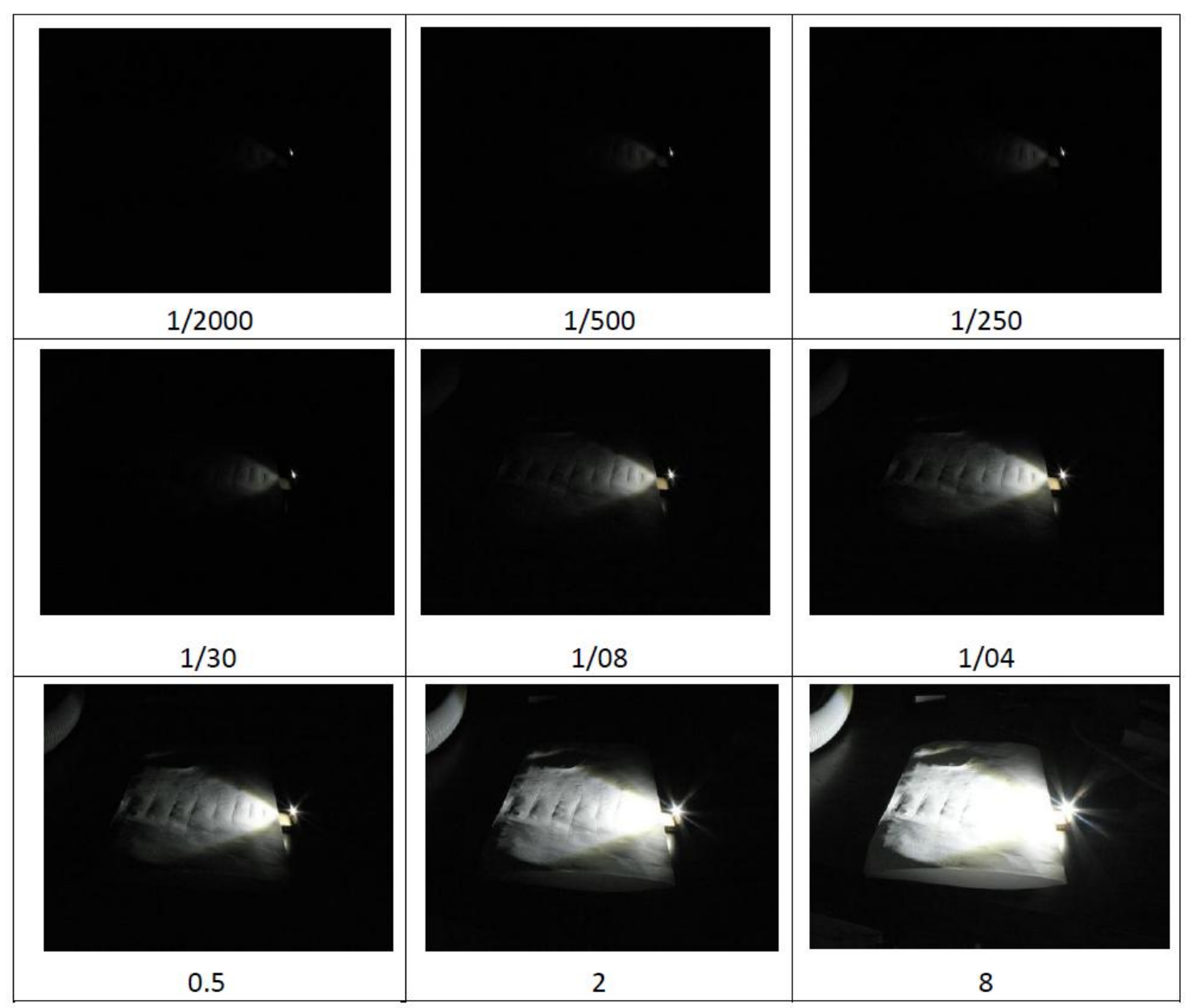

Fig. 5. Secuencia de imágenes de una lámpara encendida, con tiempos de exposición en segundos, indicados en la parte inferior de cada foto. Todas ellas con una abertura de f/8.

$$
T=2 \pi \sqrt{\frac{L}{g}}
$$

Analizando esta ecuación podemos ver que si conocemos la longitud del péndulo, y conocemos el periodo del mismo, podríamos encontrar un valor de la aceleración de la gravedad $g$, aproximado de la zona donde se realiza el experimento. Este experimento ya ha sido realizado muchas veces con gran precisión, sin embargo, nuestro objetivo será encontrar la aceleración debida a la gravedad mediante el análisis de fotografías tomadas con tiempo de exposición controlado.

\subsection{Análisis analítico}

Para nuestro análisis consideremos un péndulo simple, que consiste en una masa $M$ que cuelga de un punto fijo, a través de un hilo de longitud $L$ de masa despreciable (Fig. 6).

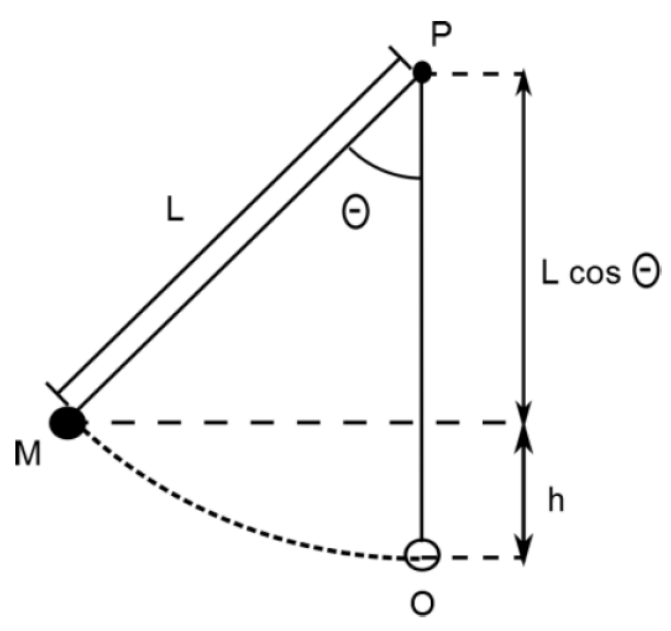

Fig. 6. Esquema de péndulo simple. 
El análisis propuesto se basa en la ley de la conservación de la energía [13], donde la energía total, sin considerar pérdidas, en cualquier instante es:

$$
E=K+U=\frac{1}{2} M L^{2} \dot{\theta}^{2}+M g L(1-\cos \theta) .
$$

Si consideramos que $\theta \ll 1$ rad., entonces $\cos \theta \approx 1-\frac{1}{2} \theta^{2}$, por lo que:

$$
E=\frac{1}{2} M L^{2} \dot{\theta}^{2}+\frac{1}{2} M g L \theta^{2} .
$$

Resolviendo para $\dot{\theta}$ observamos:

$$
\begin{gathered}
\dot{\theta}=\frac{d \theta}{d t}=\sqrt{\frac{2 E-M g L \theta^{2}}{M L^{2}}}= \\
=\sqrt{\frac{2 E}{M L^{2}}-\frac{M g L \theta^{2}}{M L^{2}}} .
\end{gathered}
$$

Con una amplitud máxima $\theta=\theta_{M}$, se designan los puntos extremos del movimiento como $\theta_{M} \mathrm{y}$ $-\theta_{M}$; en estos puntos la energía cinética es cero. Por lo tanto, $\dot{\theta}=0$ y $\theta=\theta_{M}$, así que

$$
E=\frac{1}{2} M L^{2} \theta_{M}^{2}
$$

por lo que la ecuación (4) la podemos escribir como:

$$
\frac{d \theta}{d t}=\sqrt{\frac{g}{L}} \sqrt{\theta_{M}^{2}-\theta^{2}}
$$

Ahora, si consideramos a $\theta_{i}$ como el ángulo para el cual tenemos $t=t_{i}$, e integramos:

$$
\begin{aligned}
& \int_{\theta_{i}}^{\theta_{f}} \frac{d \theta}{\sqrt{\theta_{M}^{2}-\theta^{2}}}=\sqrt{\frac{g}{L}} \int_{t_{i}}^{t_{f}} d t, \\
& g=L \frac{\left[\arcsin \left(\frac{\theta_{f}}{\theta_{M}}\right)-\arcsin \left(\frac{\theta_{i}}{\theta_{M}}\right)\right]^{2}}{\left(t_{f}-t_{i}\right)^{2}} .
\end{aligned}
$$

Es evidente que la ecuación anterior expresa la aceleración debida a la gravedad en términos de un ángulo inicial $\theta_{i}$, y de un ángulo final $\theta_{f}$.

\subsection{Diseño del experimento}

Mediante una pelota e hilo, ambos de color claro, se montó un péndulo simple, sostenido de un soporte universal y se colocó al frente de un fondo negro para contrastar y distinguir fácilmente la masa del péndulo en la fotografía.

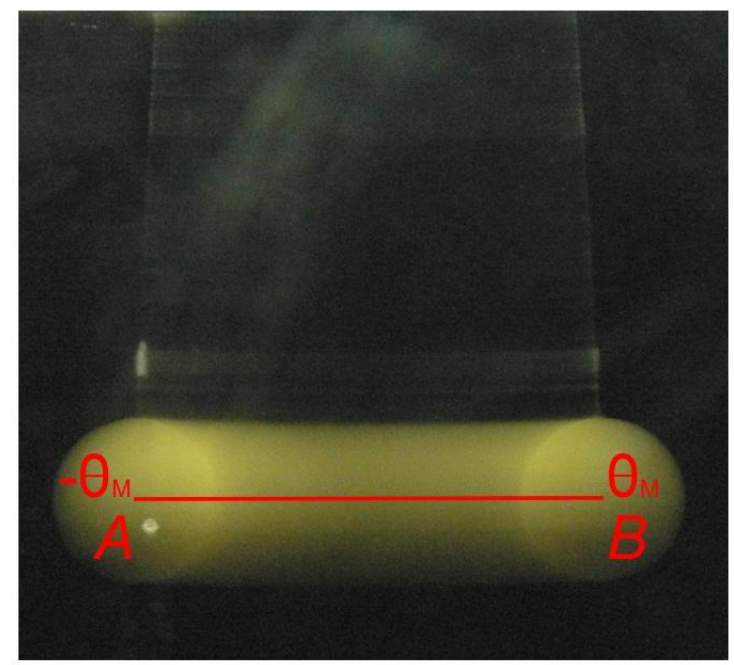

Fig. 7. Imagen de amplitud, 2.5 segundos de exposición y $f / 8.0$.

Se eligió una oscilación en un ángulo pequeño, menor a 15 grados para usar la fórmula obtenida en al análisis anterior. Las fotografías se realizaron con luz ambiente.

En nuestra experimentación se consideró un péndulo de longitud $L=(1.2300 \pm 0.0001) \mathrm{m}$. En el hilo del péndulo se dibujó un segmento arbitrario de longitud $L=(0.0500 \pm 0.0001) \mathrm{m}$, como escala para hacer conversiones entre pixeles (px) y metros (m); este segmento medido en la imagen fue igual a $(443.0 \pm 0.5)$ px. Para realizar estos análisis y conversiones, las imágenes digitales fueron tratadas con el programa libre GIMP (GNU Image Manipulation Program) [14] y editadas con el programa de computo Inkscape [15], el cual también es de licencia libre.

En la Fig. 7 se puede apreciar la oscilación completa del péndulo desde $-\theta_{M}$ hasta $\theta_{M}$, donde $\theta_{M}$ es la amplitud máxima asignada.

\subsection{Consideraciones iniciales}

Como se muestra en la Fig. 8, la cuerda $C_{o}$, que se forma por las posiciones del péndulo en los extremos, forma un triángulo isósceles con lados iguales a la longitud del péndulo $L$. El segmento formado por las posiciones del péndulo en su oscilación máxima mide $2 C_{o}=(971.0 \pm 0.5)$ px; una recta perpendicular al punto medio de la cuerda pasa por el eje de oscilación del péndulo, formando dos triángulos rectángulos. 


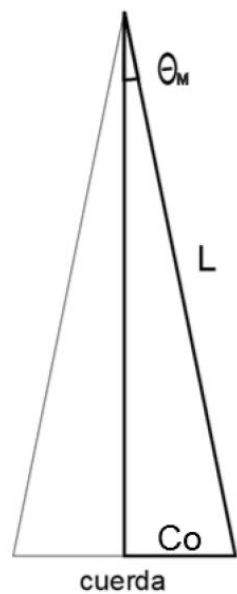

Fig. 8. Geometría empleada en el análisis de un péndulo simple.

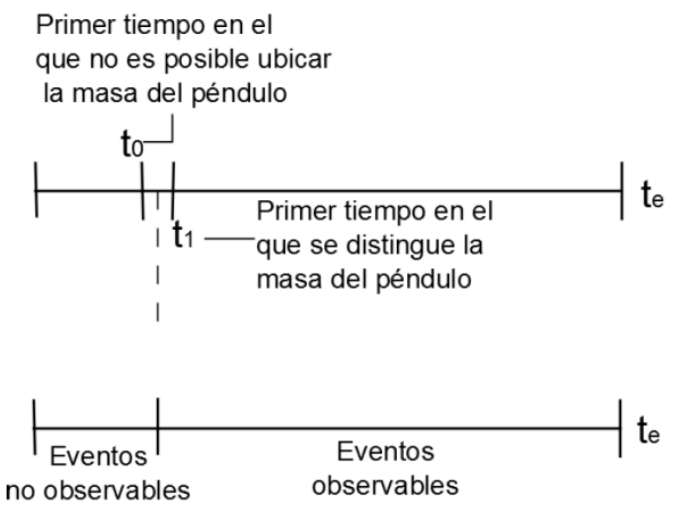

Fig. 9. Consideraciones de tiempo en la captura de las imágenes, para realizar mediciones.

Para determinar experimentalmente la amplitud de la oscilación se realizó el análisis siguiente:

$$
\sin \left(\theta_{M}\right)=\frac{C_{0}}{L}=0.0445 \pm 0.0005
$$

Debido a que se tiene un ángulo de oscilación muy pequeño, el valor de $\theta_{M}$ es aproximadamente igual a $\sin \left(\theta_{M}\right)$ (las magnitudes varían entre sí, una cantidad menor a la considerada en las cifras significativas). Entonces el ángulo de amplitud se considera:

$$
\theta_{M}=(0.0445 \pm 0.0005) \mathrm{rad} \text {. }
$$

A continuación, se tomó una fotografía con un tiempo de exposición más corto (de 1/2.5 s) para capturar un recorrido angular arbitrario. Es necesario tener cuidado de no tomar la fotografía cuando el péndulo este llegando a alguna de sus posiciones de máxima oscilación pues dificultaría determinar los ángulos inicial y final en la imagen.

Otra observación importante es que, bajo las condiciones de luz en las que se realizaron las fotografías, para tiempos de exposición menores a 1/160 s no era posible ubicar la posición del péndulo en la imagen; a partir de $1 / 125 \mathrm{~s}$ es posible distinguir la ubicación del péndulo (ver Fig. 9).

Este factor influyó en que la incertidumbre para el tiempo se considerara de la siguiente manera:

$$
t_{0}=\frac{1}{160} \mathrm{~s}, \quad t_{1}=\frac{1}{125} \mathrm{~s},
$$

y $t_{e}$ el tiempo de exposición, entonces en la imagen sólo es posible observar lo que ocurre a partir de un tiempo transcurrido entre $t_{0} \mathrm{y} t_{1}$ después de tomar la foto.

Entonces, el intervalo de tiempo $t_{r}$ en el que sucede el evento está dado por:

$$
t_{r}=t_{e}-\left(\frac{t_{0}+t_{1}}{2}\right) .
$$

Así, que el tiempo de inicio podría considerarse que se encuentra en el intervalo:

$$
\begin{aligned}
t_{i} \pm \delta t_{i}=\left(\frac{t_{0}+t_{1}}{2}\right) \pm & \left(\frac{t_{1}-t_{0}}{2}\right)= \\
& =(0.0071 \pm 0.0009) \mathrm{s} .
\end{aligned}
$$

\subsection{Resultados para obtener el valor de la aceleración $g$}

En base lo anterior y en la ecuación 1, son necesarias dos posiciones angulares adicionales y diferentes. Así que consideraremos a $\theta_{i} \mathrm{y}$ $\theta_{f}$ como posiciones arbitrarias inicial y final para un tiempo de exposición dado. Para obtener la posición angular fue necesario encontrar coordenadas de ciertos puntos útiles para el análisis. Como se mencionó anteriormente, el programa computacional Gimp maneja un plano cartesiano en pixeles con origen en la esquina superior izquierda y con dirección positiva hacia la derecha y hacia abajo.

Como ya se mencionó, primero se determinaron las coordenadas de la posición del péndulo cuando alcanza su oscilación máxima, estos se indicó en la Fig. 7. Para obtener $\theta_{i}$ y 
$\theta_{f}$ consideraremos una toma diferente (puntos $\mathrm{C}$ y D, figura 8). En esta imagen, a , se obtuvieron las coordenadas de las posiciones arbitrarias de la masa del péndulo.

A continuación, en la Fig. 11, superponemos las imágenes mencionadas, para concluir nuestro análisis. Realizando un análisis en pixeles, para después reportar en unidades métricas. En la Fig. 11 se observa cuáles son los puntos cuyas coordenadas son de interés, las posiciones A, B, C, D. Los ángulos se miden con respecto a la posición en reposo del péndulo.

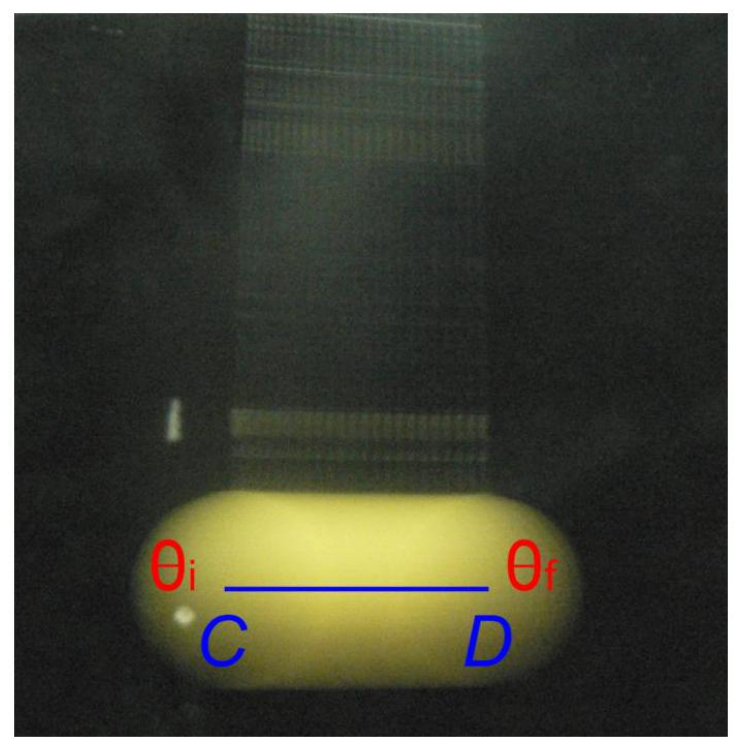

Fig. 10. Imagen de arco CD, $1 / 2.5$ segundos y $f / 2.8$.
Todas las coordenadas tienen una incertidumbre de $\pm 0.5 \mathrm{px}$ :

- $\quad$ Coordenadas de A: $(832,1058) \mathrm{px}$,

- Coordenadas de B: $(1803,1058)$ px,

- Coordenadas de C: $(934,1064) \mathrm{px}$,

- Coordenadas de D: $(1409,1064)$ px.

Con las coordenadas de A y B, se estimará la posición horizontal del punto medio, es decir, la componente horizontal de la posición en reposo del péndulo. Esto es:

$P M_{x}(A, B)=\frac{A_{x}+B_{x}}{2}=(1317.5 \pm 0.5) \mathrm{px}$,

donde $A_{x}$ y $B_{x}$ representan las coordenadas $x$ de los puntos A y B. Ahora, es necesario estimar las distancias horizontales arbitrarias de los puntos C y D, con respecto a la posición en reposo del péndulo:

$$
\begin{gathered}
d\left(C_{x}, R_{x}\right)=\left|P M_{x}-C_{x}\right|= \\
=(385.5 \pm 1.0) \mathrm{px} \\
d\left(D_{x}, R_{x}\right)=\left|P M_{x}-D_{x}\right|=(91.5 \pm 1.0) \mathrm{px} .
\end{gathered}
$$

Las distancias $d\left(C_{x}, R_{x}\right)$ y $d\left(D_{x}, R_{x}\right)$ forman dos triángulos rectángulos con hipotenusa igual a la longitud del péndulo $\theta_{i}$ y $\theta_{f}$ con ángulos $\mathrm{y}$ respectivamente, de modo que:

$$
\sin \left(\theta_{i}\right)=\frac{d\left(C_{x}, R_{x}\right)}{L}=0.0352 \pm 0.0005 .
$$
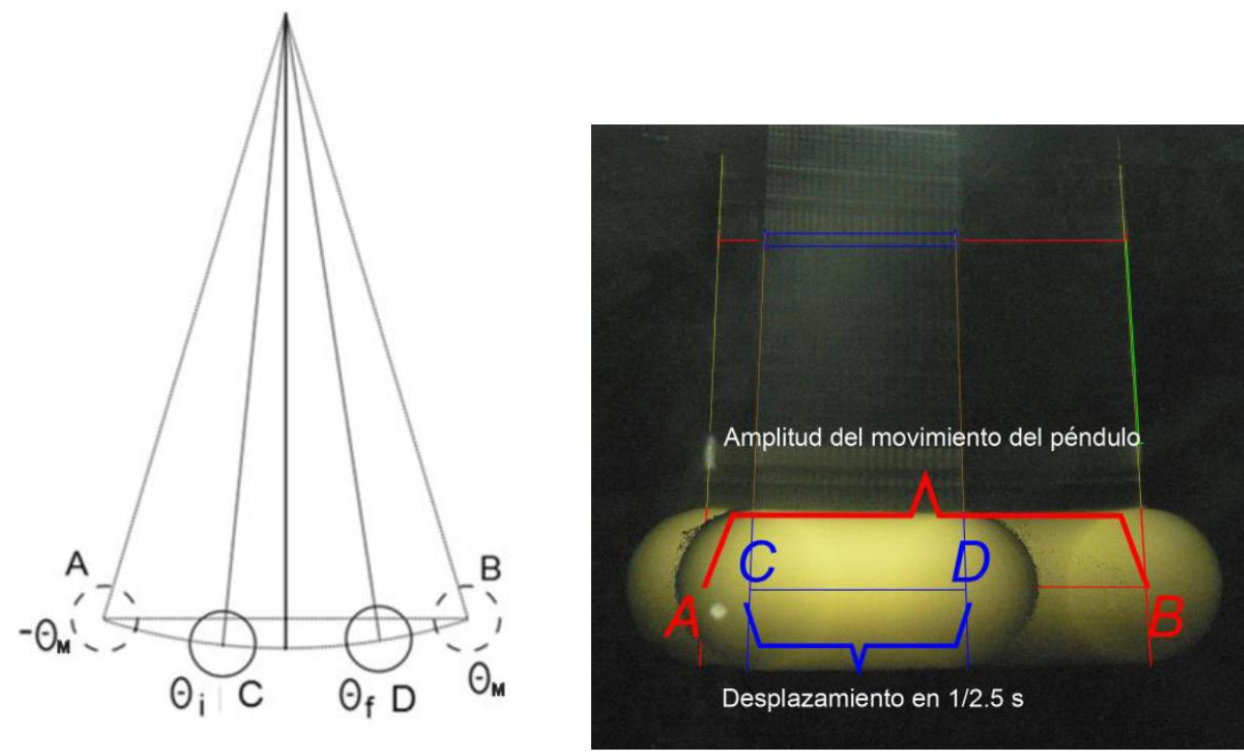

Fig. 10. Imagen de arco CD, 1/2.5 segundos y $f / 2.8$. 
Debido a que este ángulo está a la izquierda de la posición en reposo, se considera negativo, esto es:

$$
\theta_{i}=(-0.0352 \pm 0.0005) \mathrm{rad}
$$

Por otro lado:

$$
\sin \left(\theta_{f}\right)=\frac{d\left(D_{x}, R_{x}\right)}{L}=0.0084 \pm 0.0002
$$

Este ángulo abre hacia la derecha de la posición en reposo por lo que se considera positivo, esto es:

$$
\theta_{f}=(0.0084 \pm 0.0002) \mathrm{rad}
$$

De acuerdo con la Ec. (8), obtenida anteriormente, ahora se tiene la información necesaria para calcular $g$, considerando $t_{f}=t_{e}=1 / 2.5 \mathrm{~s}$, obteniéndose el resultado $g=9.6825 \mathrm{~m} / \mathrm{s}^{2}$.

La incertidumbre se determina por el método general para el cálculo de incertidumbres [16], tomando $g$ como una función de $L, t, \theta_{f} / \theta_{M} \mathrm{y}$ $\theta_{i} / \theta_{M}$ :

$$
\begin{aligned}
\delta g=\left|\frac{\partial g}{\partial L}\right| \delta L+\left|\frac{\partial g}{\partial t}\right| \delta t+ & \\
& +\left|\frac{\partial g}{\partial\left(\frac{\theta_{f}}{\theta_{M}}\right)}\right| \delta\left(\frac{\theta_{f}}{\theta_{M}}\right)+\left|\frac{\partial g}{\partial\left(\frac{\theta_{i}}{\theta_{M}}\right)}\right| \delta\left(\frac{\theta_{i}}{\theta_{M}}\right) .
\end{aligned}
$$

Tomando en cuenta las diferentes contribuciones, la incertidumbre de $g$ es igual a $\delta g=0.7435 \mathrm{~m} / \mathrm{s}^{2}$, de modo que el valor medido de la aceleración de la gravedad es:

$$
g \pm \delta g=(9.7 \pm 0.7) \mathrm{m} / \mathrm{s}^{2}
$$

El valor reportado de la aceleración de la gravedad $g$ para la ciudad de Xalapa, Veracruz (México), donde se realizaron las mediciones es de $9.78151 \mathrm{~m} / \mathrm{s}^{2}[17,18]$, lo que hace que nuestro valor medio coincida con dicho valor, dentro de los márgenes de la incertidumbre en la medida.

Como un dato adicional se puede comentar que los resultados que ofrece la fotografía digital de largo tiempo de exposición son diferentes a los que ofrece una fotografía digital convencional (tiempos estándar). En la fotografía digital de largo tiempo de exposición se captura por más tiempo la luz que es reflejada o emitida por un objeto, de modo que si este se encuentra en movimiento se podrá captar su trayectoria en una sola toma. Esta técnica solo es comparable con fotografía estroboscópica o video con cámaras de alta velocidad. La ventaja de la fotografía digital de largo tiempo de exposición es que la trayectoria deseada se observa en una sola toma, por lo que solo habrá que analizar una sola imagen y no requiere elementos adicionales o condiciones especiales, como en la fotografía estroboscópica. Por otra parte las cámaras de alta velocidad, por su costo, aun no son de fácil acceso y el análisis de las trayectorias se debe realizar de cuadro a cuadro. Finalmente, el programa computacional o software que se utilice para análisis de las imágenes es independiente de la técnica que se emplee, aquí lo importante es la información que tenga la imagen.

\section{Conclusiones}

La fotografía de largo tiempo de exposición es ampliamente utilizada con fines artísticos, sin embargo, en el laboratorio de física aun puede ser utilizada ampliamente en análisis cuantitativos.

Una vez realizadas las primeras pruebas con la fotografía de largo tiempo de exposición, fue posible concluir que esta puede ser una herramienta útil. Ello, gracias a que nos permite observar la evolución del movimiento de objetos o cuerpos, bajo condiciones normales de iluminación en laboratorio.

De nuestros resultados cuantitativos podemos concluir que con el uso de la fotografía de larga exposición nos permite obtener un valor de la aceleración de la gravedad igual a $(9.7 \pm 0.7) \mathrm{m} / \mathrm{s}^{2}$.

La fotografía de larga exposición puede ser una buena opción, por ejemplo, se puede combinar con trigonometría básica, para determinar distancias, ángulos, desplazamientos lineales y angulares, de algún objeto que se esté estudiando. Adicionalmente, esta técnica fotográfica puede emplearse para el estudio de la cinemática de los cuerpos y permite hacer un análisis muy preciso de distintos parámetros físicos.

Como se indicó, esta técnica puede ser muy útil en diferentes mediciones de los laboratorios 
de enseñanza de la física, incluso en el área de investigaciones. Algunos de los parámetros que se pueden observar son: el desplazamiento y el alcanza máximo, la distancia recorrida y los ángulos de lanzamiento. Además, contando con estos parámetros, es posible obtener otros datos del mismo movimiento, tales como: velocidades, desplazamientos angulares y tangenciales, aceleraciones, etc.

La fotografía digital de largo tiempo de exposición puede representar algunas ventajas en comparación con la fotografía estroboscópica, la cual permite también un estudio similar de los cuerpos, pero bajo condiciones controladas de luz. Contrario a lo que proponemos con las pruebas que hemos presentado, en las cuales solo se requiere nuestro objeto de estudio y una cámara digital semi-profesional o profesional, sin tener que estar muy al pendiente de las condiciones de luz en las que se lleve a cabo nuestra fotografía. Lo cual, a la vez, se convierte en una herramienta de fácil uso y de gran ayuda en el laboratorio de física.

Además de la implementación indicada en este trabajo, fue posible realizar otras pruebas para mostrar que la metodología aquí planteada es útil y efectiva. Por ejemplo, se probó para la captura de trayectorias parabólicas a diferentes ángulos iniciales, lo cual fue útil en el estudio de la Ref. [19] y para la captura de trayectorias hiperbólicas asociadas con la interferencia de dos ondas "puntuales" mecánicas, tal y como lo indica la Ref. [20]. 